\title{
Generalized Central Limit Theorem and Renormalization Group
}

\author{
Iván Calvo ${ }^{2,3 *}$, Juan C. Cuchí ${ }^{4 \dagger}$, José G. Esteve ${ }^{1,3 \ddagger}$, \\ and Fernando Falceto ${ }^{1,3} \S$ \\ ${ }^{1}$ Departamento de Física Teórica, Universidad de Zaragoza, \\ 50009 Zaragoza, Spain \\ ${ }^{2}$ Laboratorio Nacional de Fusión, Asociación EURATOM-CIEMAT, \\ 28040 Madrid, Spain \\ 3 Instituto de Biocomputación y Física de Sistemas Complejos (BIFI), \\ 50009 Zaragoza, Spain \\ ${ }^{4}$ Departament d'Enginyeria Agroforestal, Universitat de Lleida, \\ 25198 Lleida, Spain
}

\begin{abstract}
We introduce a simple instance of the renormalization group transformation in the Banach space of probability densities. By changing the scaling of the renormalized variables we obtain, as fixed points of the transformation, the Lévy strictly stable laws. We also investigate the behavior of the transformation around these fixed points and the domain of attraction for different values of the scaling parameter. The physical interest of a renormalization group approach to the generalized central limit theorem is discussed.
\end{abstract}

Keywords Mathematics: Central Limit Theorems, Stable Distributions, Characteristic Functions.

MSC2000: 60F05; 60E07; 60E10.

Keywords Physics: Renormalization Group Methods.

PACS numbers: 05.10.Cc.

\footnotetext{
*ivan.calvo@ciemat.es

${ }^{\dagger}$ cuchi@eagrof.udl.cat

‡esteve@unizar.es

${ }^{\S}$ Corresponding author. E-mail: falceto@unizar.es
} 


\section{Introduction}

The classical Central Limit Theorem states that the properly rescaled, centered sum of $n$ independent and identically distributed random variables with finite mean and variance converges to the Gaussian distribution when $n \rightarrow \infty$. This universality explains the ubiquity of the Gaussian distribution in the applications of probability theory to many branches of science. In Physics, for example, the Central Limit Theorem is behind such fundamental results like the Maxwell-Boltzmann distribution and the microscopic interpretation of diffusion by Einstein [1].

Diffusion equations are obtained as hydrodynamic limits of continuous time random walks 2] where the probability distribution of the waiting-time is Markovian with mean waiting-time $\tau$, and the probability distribution of the step-size has finite variance, $\sigma$. Therefore, the microscopic transport mechanism has finite characteristic length and time scales and the diffusion coefficient is proportional to $\sigma^{2} / \tau$. However, in the last years and in connection with the study of complex systems, it has been discovered that many processes in Physics, Biology, Economy and the Social Sciences exhibit scale-free transport [3, 4, 5], i.e. transport in which characteristic spatial and/or temporal scales are lacking. The so-called anomalous diffusion of these systems is understood in terms of non-Gaussian statistics of the underlying microscopic processes and modeled by means of fractional differential equations [6, 7]. The Generalized Central Limit Theorem [8, 9] gives all the possible limits of sums of (properly rescaled) independent and identically distributed random variables, without the hypothesis of finite variance. Precisely the limit distributions with infinite variance, usually called Lévy distributions, are the interesting ones for understanding scale-free phenomena.

On the other hand, the Renormalization Group is the main theoretical tool to investigate the universality that appears in different branches of Mathematics and Physics. Essentially, the Renormalization Group explains how a system changes when the scale of observation is modified. A Renormalization Group transformation usually consists in averaging certain degrees of freedom in a way that the original system is mapped to another with fewer degrees of freedom and different coupling constants. Such transformation defines a flow in the space of theories, with the fixed points and their linear stability properties giving much information about the large scale behavior of the system.

There is an intimate relationship between the Central Limit Theorem and the Renormalization Group. In particular, a proof of the former can be given from the perspective of the latter. This is helpful in order to understand in a different way the mechanism of convergence of the sums of distributions, which are viewed as iterations of certain Renormalization Group transformations. 
This reformulation was rigorously done by G. Jona-Lasinio [10] for the classical Central Limit Theorem, see also [11, 12, 13].

As far as we know, the aforementioned appearance of Lévy statistics in a number of transport processes is not yet well-understood from first principles. Let us focus for a moment in the observed anomalous transport in certain regimes of turbulent fusion plasmas [14, 15, 16. The conundrum can be informally posed as follows: the fundamental equations are non-linear partial differential equations (fluid momentum balance equation coupled to Maxwell equations, for example), but particle transport seems to be suitably modeled in terms of linear fractional differential equations. Let us simply mention that whereas the symbol in Fourier space of an ordinary derivative operator is $(-i k)^{n}$ with $n \in \mathbb{Z}$, the symbol of a fractional derivative operator is $(-i k)^{\alpha}$ with $\alpha \in \mathbb{R}$. A deep understanding of this change in the analyticity properties of the involved operators (or equivalently, a deep understanding of the emergence of Lévy statistics) is still lacking. From the point of view of Physics it is natural to explore the application of Renormalization Group ideas. Although a satisfactory and complete answer is probably far ahead, in the present paper we try to take a first step by showing how the Generalized Central Limit Theorem and therefore Lévy distributions show up from Renormalization Group arguments. We put special emphasis on the study of the flow in the space of probability distributions. We think that this might open a way to make further progress in the comprehension of the problems stated above.

We find it valuable to further motivate a Renormalization Group approach to the Generalized Central Limit theorem by working out rather briefly a simple but interesting physical model in which such a perspective emerges. Consider a discrete-time random walk equation

$$
n(x, t+\tau)=\int \rho(x-y) n(y, t) \mathrm{d} y
$$

where $n(x, t)$ is the density of walkers and $\rho$ is an arbitrary symmetric probability density function (p.d.f. in the following), so that $\rho(u) \mathrm{d} u$ is the probability of a step taking value in $[u, u+\mathrm{d} u]$. Jumps take place at discrete-time intervals of length $\tau$, i.e. $t=n \tau, n \in \mathbb{N}$. The so-called fluid or hydrodynamic limit of this equation is obtained by considering that the previous process occurs at a microscopic scale well-separated from the macroscopic observational space-time scales. There are at least two ways of implementing this idea.

The first way (see [17] for a clear and detailed treatment in more general random walk models) consists in relating the microscopic and macroscopic characteristic time and length by means of a scaling factor $\lambda$ that eventually goes to infinity. As long as the dimensions are properly chosen, (1.1) yields a (fractional) differential equation when $\lambda \rightarrow \infty$. Namely, define 


$$
\mathbf{n}(\mathbf{x}, \mathbf{t}):=n\left(\lambda^{\Delta} \mathbf{x}, \lambda \mathbf{t}\right)
$$

the density in macroscopic units, where $\lambda$ is the scaling factor that converts the microscopic scale into the macroscopic one. The dimension $\Delta$ has to be adjusted to obtain a non trivial fluid limit when $\lambda \rightarrow \infty$. We can write now the microscopic random walk equation (1.1) in terms of the macroscopic density

$$
\mathbf{n}(\mathbf{x}, \mathbf{t}+\tau / \lambda)=\lambda^{\Delta} \int \rho\left(\lambda^{\Delta}(\mathbf{x}-\mathbf{y})\right) \mathbf{n}(\mathbf{y}, \mathbf{t}) \mathrm{d} \mathbf{y} .
$$

Or considering its Fourier transform

$$
\hat{\mathbf{n}}(\mathbf{k}, \mathbf{t}+\tau / \lambda)=\hat{\rho}\left(\lambda^{-\Delta} \mathbf{k}\right) \hat{\mathbf{n}}(\mathbf{k}, \mathbf{t}) .
$$

If we assume

$$
\hat{\rho}(k)=1-c|k|^{\alpha}+o\left(|k|^{\alpha}\right),
$$

for some $\alpha \in(0,2], c>0$, and take $\Delta=1 / \alpha$, we obtain a non-trivial limit when $\lambda \rightarrow \infty$ :

$$
\partial_{\mathbf{t}} \hat{\mathbf{n}}(\mathbf{k}, \mathbf{t})=-\frac{c}{\tau}|\mathbf{k}|^{\alpha} \hat{\mathbf{n}}(\mathbf{k}, \mathbf{t}),
$$

that represents a diffusion equation with fractional derivatives for the density function $\mathbf{n}(\mathbf{x}, \mathbf{t})$. In particular, Einstein's derivation of the diffusion equation from a random walk model corresponds to $\alpha=2$.

Note that in the previous limit most of the details of the microscopic process have disappeared and only the lowest order in the expansion of $\hat{\rho}-1$ around $k=0$ is relevant for determining the form of the fractional diffusion equation. This is a manifestation of universality, typical in phenomena that emerge in the macroscopic domain. Observe also that the description obtained at the macroscopic level, a fractional differential equation, is of different nature to that at the microscopic level, a discrete time random walk equation.

The second way to tackle the problem is inspired by the Renormalization Group ideas. We will see that in this second approach the same description of the process, i.e. a discrete time random walk, is kept in the microscopic and macroscopic regimes. This procedure is specially useful in situations like quantum field theory where, due to the appearance of infinities, a cut-off is usually required to define the theory. To understand how this works we start by iterating (1.1) to get

$$
n(x, t+2 \tau)=\int \rho * \rho(x-y) n(y, t) \mathrm{d} y,
$$

where $\rho * \rho(x)=\int \rho(x-y) \rho(y) d y$ stands for the convolution of $\rho$ with itself. 
In order to compare this equation with the original one we perform a rescaling in space and time and introduce the new density

$$
n^{\prime}\left(x^{\prime}, t^{\prime}\right)=n\left(a x^{\prime}, 2 t^{\prime}\right), \quad a>0 .
$$

In terms of this density Eq. (1.6) reads

$$
n^{\prime}\left(x^{\prime}, t^{\prime}+\tau\right)=\int T_{a} \rho\left(x^{\prime}-y^{\prime}\right) n^{\prime}\left(y^{\prime}, t^{\prime}\right) \mathrm{d} y^{\prime}
$$

that looks exactly like (1.1) except that the p.d.f. has changed to

$$
T_{a} \rho(x)=a \rho * \rho(a x) .
$$

We call $T_{a}$ the renormalization group transformation.

If we examine carefully what (1.7) means, we observe that although it looks like a process in discrete time $\tau$ with p.d.f. $T_{a} \rho$, it actually represents the original process with p.d.f. $\rho$, discrete time $\tau / 2$ and rescaled space variable. The fluid or diffusive limit is obtained when we apply this procedure infinitely many times, so that we can write

$$
\mathbf{n}(\mathbf{x}, \mathbf{t}+\tau)=\int \boldsymbol{\rho}(\mathbf{x}-\mathbf{y}) \mathbf{n}(\mathbf{y}, \mathbf{t}) \mathrm{d} \mathbf{y} .
$$

where

$$
\boldsymbol{\rho}=\lim _{m \rightarrow \infty} T_{a}^{m} \rho
$$

Therefore, in order to have a well defined process in the fluid limit we should adjust $a$ so that the previous limit exists. $\rho$ is necessarily a fixed point of the renormalization group transformation. Universality in this approach shows up because the fluid limit is the same for all initial p.d.f.'s $\rho$ that belong to the domain of attraction of the same fixed point under the action of $T_{a}$. All these considerations motivate the interest of the study of the renormalization group transformation $T_{a}$, its fixed points and their stability.

The rest of the paper is organized as follows. In Section 2 we introduce more precisely the renormalization group transformation and study the behavior of the moments. In Section 3 we establish the appropriate differential setup for investigating topological properties of the transformation. Finally, Section 4 is devoted to the study of the fixed points of the transformation, their stability, and their domain of attraction.

\section{Renormalization group transformation}

Let $\rho$ be a p.d.f. in $\mathbb{R}$, i.e. 
(i) $\rho(x) \geq 0, \forall x \in \mathbb{R}$ and

(ii) $\int_{-\infty}^{\infty} \rho(x) \mathrm{d} x=1$;

we define the following Renormalization Group transformation acting on it

$$
T_{a} \rho(x):=|a| \int_{-\infty}^{\infty} \rho(a x-s) \rho(s) \mathrm{d} s,
$$

where $a$ is a real number different from zero. Note that for normalized $\rho, T_{a} \rho$ is also normalized.

The meaning of the transformation is clear; $T_{a} \rho$ is the probability density of the random variable $\xi^{\prime}=\left(\xi_{1}+\xi_{2}\right) / a$ where $\xi_{1}, \xi_{2}$ are independent identically distributed random variables with density $\rho$. The two essential ingredients of the renormalization group: new variables that represent an average of the old ones and the scaling of the former, are present in this example in the simplest way.

To understand how the different values of $a$ lead to different properties of the transformation it is instructive to consider the situation in which all moments

$$
\left\langle x^{n}\right\rangle_{\rho}=\int_{-\infty}^{\infty} x^{n} \rho(x) \mathrm{d} x, \quad n \in \mathbb{N},
$$

are finite (in the subsequent sections we will work out the general case) and study their behavior under the transformation. One immediately obtains the following expression for the moments of the transformed probability distribution:

$$
\left\langle x^{n}\right\rangle_{T_{a} \rho}=a^{-n} \sum_{i=0}^{n}\left(\begin{array}{c}
n \\
i
\end{array}\right)\left\langle x^{i}\right\rangle_{\rho}\left\langle x^{n-i}\right\rangle_{\rho}, \quad n \in \mathbb{N} .
$$

If we take in particular $n=1$ we get:

$$
\langle x\rangle_{T_{a} \rho}=\frac{2}{a}\langle x\rangle_{\rho}
$$

which implies that the transformation maps densities with zero mean into themselves. In this case the relation between the variances is

$$
\left\langle x^{2}\right\rangle_{T_{a} \rho}=\frac{2}{a^{2}}\left\langle x^{2}\right\rangle_{\rho}
$$

Now, we can distinguish three different regimes after $m$ successive applications of the transformation $T_{a}$ according to the values of $a$, when $m \rightarrow \infty$ :

- For $|a|>\sqrt{2}$

$$
\lim _{m \rightarrow \infty}\left\langle x^{2}\right\rangle_{T_{a}^{m} \rho}=0
$$


which implies that for distributions with finite variance, $T_{a}^{m} \rho$ should approach the Dirac delta function in the large $m$ limit. In the next section we shall make precise the topological space in which $T_{a}$ acts and we shall see in which sense the above limit holds.

- For $|a|<\sqrt{2}$, assuming that $\left\langle x^{2}\right\rangle_{\rho} \neq 0$, we have

$$
\lim _{m \rightarrow \infty}\left\langle x^{2}\right\rangle_{T_{a}^{m} \rho}=\infty \text {. }
$$

- Finally, when $|a|=\sqrt{2}$, the value of $\left\langle x^{2}\right\rangle$ does not change under the application of the renormalization group transformation. In this case we have a fixed point with finite non-zero moments. Denoting by $\rho_{0}$ the fixed point, $T_{a} \rho_{0}=\rho_{0}$, we will have that

$$
\left\langle x^{2 n}\right\rangle_{\rho_{0}}=\frac{1}{2^{n}-2} \sum_{i=1}^{n-1}\left(\begin{array}{c}
2 n \\
i
\end{array}\right)\left\langle x^{2 i}\right\rangle_{\rho_{0}}\left\langle x^{2(n-i)}\right\rangle_{\rho_{0}},
$$

which is solved by

$$
\left\langle x^{2 n}\right\rangle_{\rho_{0}}=\left(\left\langle x^{2}\right\rangle_{\rho_{0}}\right)^{n} \frac{(2 n) !}{n ! 2^{n}} .
$$

That solution coincides, of course, with the expression for the moments of the Gaussian distribution.

\section{Banach space structure}

The natural framework to ask topological and differential questions about $T_{a}$ is that of Banach spaces. In our case we shall consider test functions in $C_{\infty}(\mathbb{R})$ : the Banach space of continuous functions with vanishing limit at $\infty$, endowed with the supremum norm. The probability densities are the positive distributions of unit norm in its topological dual $C_{\infty}(\mathbb{R})^{\prime}$. The space $C_{\infty}(\mathbb{R})^{\prime}$ consists of the finite, complex Radon measures in $\mathbb{R}$; it contains, for instance, distributions supported on discrete sets (Dirac delta functions) as well as densities in $L^{1}(\mathbb{R})$.

We shall denote the action of an element of $\rho \in C_{\infty}(\mathbb{R})^{\prime}$ on $f \in C_{\infty}(\mathbb{R})$, $\rho f$, by

$$
\rho f:=\int_{-\infty}^{\infty} \rho(x) f(x) \mathrm{d} x .
$$

It is a classical result, see [20] for instance, that the convolution $\rho_{1} * \rho_{2}$ of two finite Radon measures is again a finite Radon measure and actually $C_{\infty}(\mathbb{R})^{\prime}$ is a commutative Banach algebra with the convolution. In particular, the convolution is associative and satisfies

$$
\left\|\rho_{1} * \rho_{2}\right\| \leq\left\|\rho_{1}\right\|\left\|\rho_{2}\right\|,
$$


where the equality is attained if both distributions are positive.

To define the renormalization group transformation in $C_{\infty}(\mathbb{R})^{\prime}$ we introduce the dilation $\pi_{r}: C_{\infty}(\mathbb{R}) \rightarrow C_{\infty}(\mathbb{R})$,

$$
\left(\pi_{r} f\right)(x):=f(r x)
$$

Now we can obtain the transformed distribution under the renormalization group by means of the formula

$$
T_{a} \rho=(\rho * \rho) \pi_{1 / a}
$$

that correctly defines a linear functional $T_{a} \rho: C_{\infty}(\mathbb{R}) \rightarrow \mathbb{R}$.

The main differential properties of the renormalization group transformation are collected in the following proposition.

\section{Proposition 1:}

The transformation $T_{a}: C_{\infty}(\mathbb{R})^{\prime} \rightarrow C_{\infty}(\mathbb{R})^{\prime}$ in (3.1) is continuous and differentiable with bounded continuous differential.

Proof: To show the continuity of $T_{a}$ it is enough to realize that $\pi_{1 / a}$ is an isometry. Then, we get

$$
\begin{aligned}
\left\|T_{a} \rho_{1}-T_{a} \rho_{2}\right\| & =\left\|\left(\rho_{1}+\rho_{2}\right) *\left(\rho_{1}-\rho_{2}\right) \pi_{1 / a}\right\| \\
& \leq\left\|\rho_{1}+\rho_{2}\right\|\left\|\rho_{1}-\rho_{2}\right\|,
\end{aligned}
$$

from which the continuity of $T_{a}$ follows. On the other hand, the differential of $T_{a}$ is given by

$$
\left(D T_{a}\right)_{\rho} \zeta=2(\rho * \zeta) \pi_{1 / a}
$$

which is a bounded linear map. In fact one has

$$
\left\|\left(D T_{a}\right)_{\rho} \zeta\right\|=2\|\rho * \zeta\| \leq 2\|\rho\|\|\zeta\| .
$$

Therefore,

$$
\left\|\left(D T_{a}\right)_{\rho}\right\| \leq 2\|\rho\|,
$$

and the equality is attained if $\rho$ is positive. As for the continuity of the differential simply observe that

$$
\left\|\left(D T_{a}\right)_{\rho_{1}}-\left(D T_{a}\right)_{\rho_{2}}\right\|=\left\|\left(D T_{a}\right)_{\rho_{1}-\rho_{2}}\right\| \leq 2\left\|\rho_{1}-\rho_{2}\right\|,
$$

and the proof is concluded. 


\section{Fixed points}

Much information about the renormalization group transformation is obtained by studying its fixed points and its linearization around them. In order to work

out the fixed points it is convenient to express the transformation in terms of the characteristic functions

$$
\widehat{T_{a} \rho}(k)=\int T_{a} \rho(x) \mathrm{e}^{i k x} d x=\widehat{\rho}(k / a)^{2} .
$$

Then, for the fixed point $T_{a} \rho_{0}=\rho_{0}$, one has

$$
\widehat{\rho}_{0}(k / a)^{2}=\widehat{\rho}_{0}(k) .
$$

Note that this equation is solved by functions $\widehat{\rho}$ whose logarithm is a symmetric, homogeneous function of degree $\alpha=\log 2 / \log |a|$. To find more general solutions we distinguish two cases according to the sign of $a$.

- If $a$ is positive, we have solutions for (4.1) of the form

$$
\widehat{\rho}_{0}(k)=\widehat{S}_{\alpha, A}(k):=\exp \left(-A|k|^{\alpha} \theta(k)-\bar{A}|k|^{\alpha} \theta(-k)\right),
$$

where $\alpha$ is given by the relation $|a|=2^{1 / \alpha}, \bar{A}$ is the complex conjugate of $A$ and $\theta$ is the Heaviside step function.

The fact that $\widehat{S}_{\alpha, A}$ is the characteristic function of a probability density (i.e. a positive functional) imposes additional restrictions on $A$ and $\alpha$. In particular it is well-known (see Refs. [8, 9] for instance) that if $A \neq 0$, then we must have

$$
0<\alpha \leq 2 \quad \text { and } \quad A=|A| \mathrm{e}^{i \varphi} \quad \text { with } \quad|\varphi| \leq \frac{\pi}{2}(1-|\alpha-1|) .
$$

The solution with $A=0$ defines a fixed point of the renormalization group, corresponding to the Dirac delta function, that exists for any value of $a$.

These solutions of (4.1) are the so-called strictly stable laws, denoted here by $S_{\alpha, A}$, that where introduced by P. Lévy [8], see also [9, 18, 19].

- For negative $a$, the positive and negative values of $k$ are related through (4.1). As a result, the fixed points are the ones described above for $|a|$ but restricted to real values of $A$.

After discussing the fixed points of our renormalization group transformation we analyze their stability and the domain of attraction (around the fixed point). The standard way to approach this problem is to study the spectrum 
and eigenvectors of the differential of the transformation at the fixed points. The results can be summarized in the following

\section{Proposition 2:}

Let $\left(D T_{a}\right)_{\rho}$ be the operator defined in (3.3), $\rho_{0}$ one of the fixed points of $T_{a}$ discussed above, and $\boldsymbol{\sigma}$ the complex spectrum of $\left(D T_{a}\right)_{\rho_{0}}$.

(i) If $\rho_{0}=S_{\alpha, A}$ with $\operatorname{Re} A>0$, then $\sigma=\{\lambda$, s. $t$. $|\lambda| \leq 2\}$ and all its values belong to the pointwise spectrum except for $\lambda=0$.

(ii) If $\rho_{0}=S_{\alpha, A}$ with $\operatorname{Re} A=0$, then $\sigma=\{\lambda$, s. t. $|\lambda|=2\}$ with its only eigenvalue at $\lambda=2$.

Proof: In order to determine the pointwise spectrum one has to solve the eigenvalue equation

$$
\left(D T_{a}\right)_{\rho_{0}} \zeta=\lambda \zeta
$$

We will focus first on (i), corresponding to the non-trivial fixed point $\rho_{0}=$ $S_{\alpha, A}$ with $\operatorname{Re} A>0$. The equation for the eigenvalues (4.3) can be more easily handled by writing it in terms of the characteristic functions, $\widehat{\zeta}$. Namely,

$$
2 \widehat{S}_{\alpha, A}(k / \alpha) \widehat{\zeta}(k / \alpha)=\lambda \widehat{\zeta}(k) .
$$

Using the ansatz $\widehat{\zeta}(k)=\widehat{S}_{\alpha, A}(k) \widehat{\eta}(k)$ and taking into account the properties of the fixed point, we arrive at the following equation

$$
2 \widehat{\eta}(k / a)=\lambda \widehat{\eta}(k),
$$

which can be solved with homogeneous functions of the appropriate degree. Namely, for positive $a=2^{1 / \alpha}$ we have solutions of the form

$$
\widehat{\eta}_{s}(k)=\left(B_{+} \theta(k)+B_{-} \theta(-k)\right)|k|^{s}
$$

with eigenvalue $\lambda_{s}=2^{1-\frac{s}{\alpha}}$.

In order to ensure that $\zeta=S_{\alpha, A} * \eta \in C_{\infty}(\mathbb{R})^{\prime}$ we must have Re $s \geq 0$. We shall now show that it is also sufficient. The function

$$
\zeta_{s}(x)=\frac{1}{2 \pi} \int_{-\infty}^{\infty} \widehat{\eta}_{s}(k) \mathrm{e}^{-A|k|^{\alpha} \theta(k)-\bar{A}|k|^{\alpha} \theta(-k)} \mathrm{e}^{-i k x} \mathrm{~d} k,
$$

where $\operatorname{Re} A>0$ and $\operatorname{Re} s \geq 0$, is clearly continous and even smooth, so we only need to show that it decays sufficiently fast for large $|x|$. Consider the integration of the positive momenta 


$$
\zeta_{+}(x)=\frac{B_{+}}{2 \pi} \int_{0}^{\infty} k^{s} \mathrm{e}^{-A k^{\alpha}-i k x} \mathrm{~d} k .
$$

Assuming that $x>0$ and $\operatorname{Re} A>0$ we can perform a slight clockwise rotation of angle $\gamma$ of the integration line in the complex plane. It does not affect the result due to Cauchy's theorem. If we also change to a new variable $z=\mathrm{e}^{i \gamma} k x$ the integral reads

$$
\zeta_{+}(x)=\frac{B_{+} \mathrm{e}^{-i \gamma(s+1)}}{2 \pi x^{s+1}} \int_{0}^{\infty} z^{s} \mathrm{e}^{-A(z / x)^{\alpha} \mathrm{e}^{-i \alpha \gamma}-i z \mathrm{e}^{-i \gamma}} \mathrm{d} z
$$

and for small $\gamma>0$ we can safely take the limit of large $x$ inside the integral to get

$$
\zeta_{+}(x)=\frac{f(x)}{x^{s+1}}, \quad \text { with } \quad \lim _{x \rightarrow \infty} f(x)=B_{+} \frac{\Gamma(s+1)}{2 \pi} \mathrm{e}^{-i \pi(s+1) / 2} .
$$

We can deal in an analogous way with the integration on the negative semiaxis and also with the case of negative $x$. In all cases we can show that provided $\operatorname{Re} s>0$ the function decays fast enough so that $\zeta=S_{\alpha, A} * \eta \in C_{\infty}(\mathbb{R})^{\prime}$

In this way we prove that all values of $\lambda$ s.t. $0<|\lambda| \leq 2$ are in the pointwise spectrum. As the spectrum must be a closed set, 0 is also in $\boldsymbol{\sigma}$. To exclude other points, i.e $|\lambda|>2$ we can use the well-known argument that if $|\lambda|>\left\|\left(D T_{a}\right)_{\rho_{0}}\right\|=2$ then it belongs to the resolvent. This concludes the proof of part (i) of the proposition.

We proceed to prove part (ii). There are only two possibilities of having a fixed point $S_{\alpha, A}$ with $\operatorname{Re} A=0$ : either $\alpha=1$ and $\varphi= \pm \pi / 2$ or $A=0$. In all other instances $\operatorname{Re} A>0$. We shall discuss in detail the fixed point with $A=0$, i.e. the Dirac delta function $\delta$, which exists for any value of $a$. To study the spectrum of the differential at this point we introduce the operator

$$
U \zeta:=\frac{1}{2}\left(D T_{a}\right)_{\delta} \zeta=\zeta \pi_{1 / a}
$$

$U$ is an isometry and, therefore, its spectrum lies entirely in the unit circle. On the other hand the equation for the eigenvalues

$$
U \zeta_{\mu}=\mu \zeta_{\mu}
$$

is solved with $\mu=1$ and $\zeta_{1}=\delta$. To exclude the possibility of any other solution we can use the following simple argument (valid for $a=2^{1 / \alpha}>1$, although a minor modification allows to deal with the case $a<-1$ ).

Consider a function $\phi \in C_{\infty}(\mathbb{R})$ with $\phi(a)=\bar{\mu} \phi(1)$ and $\|\phi\|=1$, but arbitrary otherwise. Now for any positive integer $N$ we construct the function $f^{(N)} \in C_{\infty}(\mathbb{R})$ defined for $x \in\left[a^{-N}, a^{N}\right]$ by

$$
f^{(N)}\left(a^{n} x\right)=\bar{\mu}^{n} \phi(x), \quad x \in[1, a], \quad n=-N,-N+1, \ldots, N-1, N,
$$


and extended to $\mathbb{R}$ so that $\left\|f^{(N)}\right\|=1$. Thus,

$$
\begin{aligned}
\int_{w a^{n}}^{w a^{n+1}} \zeta_{\mu}(x) f^{(N)}(x) \mathrm{d} x & =\int_{w a^{n+1}}^{w a^{n+2}} \zeta_{\mu}(x) f^{(N)}(x) \mathrm{d} x=I_{w}, \\
w & \in(1, a), \quad n=-N, \ldots, N-3 .
\end{aligned}
$$

Then

$$
\int_{w a^{-N}}^{w a^{N-1}} \zeta_{\mu}(x) f^{(N)}(x) \mathrm{d} x=(2 N-1) I_{w}
$$

and, if $I_{w} \neq 0, \zeta_{\mu}$ is an unbounded functional. Therefore $I_{w}=0$, implying that the intersection of the support of $\zeta_{\mu}$ with $(0, \infty)$ is empty. A similar argument rules out the negative semiaxis and finally one deduces that $\zeta_{\mu}$ must be supported in $\{0\}$, i.e. it is the $\delta$-function that corresponds to $\mu=1$.

As for the rest of the spectrum consider, for $\mu=\mathrm{e}^{i \theta}$ and a positive integer $K$, the following distribution $\zeta_{\mu}^{(K)} \in C_{\infty}^{\prime}(\mathbb{R})$ :

$$
\zeta_{\mu}^{(K)}(x)= \begin{cases}0 & \text { if }|x| \notin(1 / K, K), \\ \frac{1}{4 \log K} \frac{\mathrm{e}^{i \theta \log |x| / \log |a|}}{|x|} & \text { if }|x| \in(1 / K, K) .\end{cases}
$$

One can compute $\left\|\zeta_{\mu}^{(K)}\right\|=1$ and

$$
\left\|U \zeta_{\mu}^{(K)}-\mu \zeta_{\mu}^{(K)}\right\|=\frac{\log a}{\log K},
$$

which shows that $\mu=\mathrm{e}^{i \theta}$ is in the spectrum of $U$. Therefore, the complex spectrum of $\left(D T_{a}\right)_{\delta}=2 U$ is as stated in the second part of the proposition.

As mentioned before, the only other possibility to obtain a fixed point with $\operatorname{Re} A=0$ is having $\alpha=1$ and $\varphi= \pm \pi / 2$. The fixed point in this case is $\delta(x+e)$ with $e=\operatorname{Im} A$. The proof above can be applied to this case by simply shifting to $-e$ the origin of the real line.

We would like to discuss now the information about the behavior of the renormalization group transformation around the fixed points that can be extracted from the study of the spectrum. We start by considering the fixed points corresponding to strictly stable Lévy distributions. In this case the stable directions that lie on the domain of attraction of the fixed point correspond to $\left|\lambda_{s}\right|<1$, i.e. $s>\alpha$, while those with $s<\alpha$ give $\left|\lambda_{s}\right|>1$ and therefore unstable perturbations of the fixed point.

It is interesting to analyze the behavior of the characteristic function of the fixed point perturbed in the $\zeta_{s}$ direction for small values of $k$. First note that

$$
\widehat{\rho}_{0}(k)=1-A|k|^{\alpha} \theta(k)-\bar{A}|k|^{\alpha} \theta(-k)+o\left(|k|^{\alpha}\right)
$$


and that

$$
\widehat{\rho}_{0}(k)+\epsilon \widehat{\zeta}_{s}(k)= \begin{cases}1-A|k|^{\alpha} \theta(k)-\bar{A}|k|^{\alpha} \theta(-k)+o\left(|k|^{\alpha}\right) & \text { for } s>\alpha, \\ 1+B_{+} k^{s} \theta(k)+B_{-}|k|^{s} \theta(-k)+o\left(|k|^{s}\right) & \text { for } s<\alpha,\end{cases}
$$

which implies that the perturbations that do not change the behavior of the characteristic function around $k=0$ are precisely the stable directions. In this way we obtain an infinitesimal version of the well-known result [21] that the the p.d.f.s whose characteristic function behaves around $k=0$ as

$$
\widehat{\rho}(k)=1-A|k|^{\alpha} \theta(k)-\bar{A}|k|^{\alpha} \theta(-k)+o\left(|k|^{\alpha}\right)
$$

belong to the domain of attraction of the fixed point with characteristic function

$$
\widehat{\rho}_{0}(k)=\exp \left(-A|k|^{\alpha} \theta(k)-\bar{A}|k|^{\alpha} \theta(-k)\right) .
$$

We consider now the other fixed point, the Dirac delta function. From the results of Section 2 (see also below) one would expect to have an attractive fixed point at $\delta$, at least for certain values of $a$. Therefore, it may seem surprising that the spectrum of the differential around the fixed point $\delta$ has only eigenvalues with modulus greater than 1 . The reason is that the eigenvectors with $|\lambda|<1$ are associated to the strong convergence of the iterated transformation of the fixed point perturbed in that direction, while in the case of $\delta$, strong convergence is impossible and we have convergence only in the weak topology. This fact can be shown by looking more closely at the behavior of the transformation around the fixed point. Consider a perturbation of $\delta$ by $h \in C_{\infty}^{\prime}(\mathbb{R})$ with $0<\|h\|<1$. Then

$$
\begin{aligned}
\left\|T_{a}(\delta+h)-\delta\right\| & =\|2 h+h * h\| \geq \\
& \geq 2\|h\|-\|h * h\| \geq 2\|h\|-\|h\|^{2}>\|h\|,
\end{aligned}
$$

and all directions around the fixed point $\delta$ are unstable in the strong topology. The situation in the weak topology is different and, as shown below, there are p.d.f.s in $L^{1}$ which under successive application of the renormalization group transformation converge weakly to the Dirac delta function.

In order to complete the local picture obtained above and to substantiate previous statements, we shall discuss the behavior of the iteration of the transformation for different initial densities and different values of $a$. Let

$$
\widehat{\rho}(k)=1-B|k|^{\nu} \theta(k)-\bar{B}|k|^{\nu} \theta(-k)+o\left(|k|^{\nu}\right),
$$

with $0<\nu \leq 2$ and $B \neq 0$. Therefore, for fixed $k$ and $a=2^{1 / \alpha}>1$ one has

$$
\widehat{T_{a}^{n}} \rho(k)=\left(1-a^{-\nu n} B|k|^{\nu} \theta(k)-a^{-\nu n} \bar{B}|k|^{\nu} \theta(-k)+o\left(a^{-\nu n}\right)\right)^{2^{n}}
$$


and the limit of large $n$ with $k \neq 0$ reads

$$
\lim _{n \rightarrow \infty} \widehat{T_{a}^{n} \rho}(k)= \begin{cases}1 & \text { for } \nu>\alpha, \\ \exp \left(-B|k|^{\nu} \theta(k)-\bar{B}|k|^{\nu} \theta(-k)\right) & \text { for } \nu=\alpha, \\ 0 & \text { for } \nu<\alpha\end{cases}
$$

We therefore have the following proposition:

\section{Proposition 3:}

Take $\rho \in C_{\infty}^{\prime}(\mathbb{R})$ with characteristic function given by $(4.10)$ and $a=2^{1 / \alpha}$. Then, $T_{a}^{n} \rho$ converges weakly when $n \rightarrow \infty$ to $\delta$ if $\nu>\alpha$, to $S_{\alpha, B}$ if $\nu=\alpha$ and it does not converge if $\nu<\alpha$.

The result is a simple consequence of Lévy's continuity theorem together with the behavior of the characteristic functions in (4.11).

With a similar computation we obtain for $a=-2^{1 / \alpha}<-1$ the following:

- If $\nu>\alpha, T_{a}^{n} \rho$ converges weakly to the $\delta$ function.

- For $\nu=\alpha$ we have a limit two-cycle formed by the strictly stable densities $S_{\alpha, B}$ and $S_{\alpha, \bar{B}}$. The two members of the cycle are related by reflection with respect to the origin $x \mapsto-x$.

- Finally, for $\nu<\alpha$ the sequence does not converge.

In order to illustrate some of the advantages of our approach to the Generalized Central Limit Theorem we will give now a simple proof, based on the above results, of the positivity of $S_{\alpha, A}$ for $\alpha$ and $A$ as in (4.2). The proof goes as follows:

Take the following p.d.f.

$$
\rho(x)=\frac{c_{1}}{n^{\alpha+1}+|x|^{\alpha+1}} \theta(-x)+\frac{c_{2}}{n^{\alpha+1}+|x|^{\alpha+1}} \theta(x),
$$

with $c_{i} \geq 0, c_{1}+c_{2}>0, \alpha \in(0,1)$. $n$ is a positive real number, chosen so that $\|\rho\|=1$. Standard estimates show that

$$
\widehat{\rho}(k)=1-A|k|^{\alpha} \theta(k)-\bar{A}|k|^{\alpha} \theta(-k)+o\left(|k|^{\alpha}\right),
$$

where $A=|A| \mathrm{e}^{i \phi}$ with

$$
|A|=\left(c_{1}^{2}+c_{2}^{2}+2 c_{1} c_{2} \cos (\pi \alpha)\right)^{1 / 2} \frac{\Gamma(1-\alpha)}{\alpha}
$$


and

$$
\tan \phi=\frac{c_{2}-c_{1}}{c_{1}+c_{2}} \tan \left(\frac{\pi \alpha}{2}\right) \quad \text { with }|\phi| \leq \frac{\pi}{2} .
$$

On the other hand, from our previous results we have

$$
\lim _{n \rightarrow \infty} T_{a}^{n} \rho=S_{\alpha, A},
$$

which, due to the fact that our transformation preserves positivity, implies that $S_{\alpha, A}$ is positive. Note that taking different non-negative values for $c_{1}$ and $c_{2}$ we cover the whole range for $A$ as described in (4.2).

A slight modification of the initial p.d.f. and the estimate allows to deal with the remaining cases, i.e. $\alpha \in[1,2]$.

\section{Conclusions}

In this paper we have studied a simple instance of the renormalization group transformation on the space of probability densities. We have shown that by changing the scaling that governs the transformation one obtains different fixed points or limit two-cycles that correspond to the strictly stable laws of Lévy and the limiting case of the Dirac $\delta$ distribution.

We have also studied the stability of the fixed points using the linear approximation of the transformation around the fixed point. In this way we have derived a local version of the classical results about the domain of attraction

of the stable laws. We have also shown that the stable or unstable character of the fixed point can be different if we consider the strong or the weak topology in the space of probability densities.

Acknowledgements: We wish to thank the referees for a careful reading and useful comments that helped us to improve the paper. I. C. and J. C. C. gratefully acknowledge the hospitality of the Department of Theoretical Physics at the University of Zaragoza, where part of this work was done. Research partially supported by grant FIS2006-01225 MEC (Spain), and grants ENE200907247 and FPA2009-09638, Ministerio de Ciencia e Innovación (Spain).

\section{References}

[1] A. Einstein, Ann. Phys. 17, 549 (1905).

[2] E. W. Montroll and G. Weiss, J. Math. Phys. 6, 167 (1965).

[3] J.-P. Bouchaud and A. Georges, Phys. Rep. 195, 127 (1990). 
[4] R. Metzler and J. Klafter, Phys. Rep. 339, 1 (2000).

[5] G. Zaslavsky, Phys. Rep. 371, 461 (2002).

[6] H. C. Fogedby, Phys. Rev. E 50, 1657 (1994).

[7] I. Podlubny, Fractional Differential Equations, Academic Press, San Diego, (1999).

[8] P. Lévy, Théorie de l'addition des variables aléatoires, Gauthiers-Villars (1937).

[9] B. V. Gnedenko and A. N. Kolmogorov, Limit distributions for sums of independent random variables, Addison-Wesley (1954).

[10] G. Jona-Lasinio, Nuovo Cimento 26, 98 (1975).

[11] J. Honkonen, Phys. Rev. E 53, 327 (1996).

[12] G. Jona-Lasinio, Phys. Rep. 352, 439 (2001).

[13] L. Koralov and Y. G. Sinai, Theory of probability and Random Processes, Springer (2007).

[14] D. del-Castillo-Negrete, B. A. Carreras and V. E. Lynch, Phys. Rev. Lett. 94, 065003 (2005).

[15] J. A. Mier, R. Sánchez, L. García, D. E. Newman, and B. A. Carreras, Phys. Plasmas 15, 112301 (2008).

[16] R. Sánchez, D. E. Newman, J.-N. Leboeuf, V. K. Decyk and B. A. Carreras, Phys. Rev. Lett. 101, 205002 (2008).

[17] E. Scalas, R. Gorenflo and F. Mainardi, Phys. Rev. E 69, 011107 (2004).

[18] I. A. Ibragimov and Y. V. Linnik, Independent and Stationary Sequences of Random Variables, (J. F. C. Kingman, ed.) Wolters-Noordhoff (1971).

[19] J. Aaronson and M. Denker, Ann. Probab. 26, 399-415 (1998).

[20] W. Rudin, Real and Complex Analysis, McGraw-Hill (1987).

[21] B. V. Gnedenko, and V. S. Koroluk, Dopov. Nats. Akad. Nauk Ukraïni 4, 275-278 (1950). 